\title{
Excesso de peso, obesidade e educação no Brasil
}

\author{
Overweight, obesity and education in Brazil
}

Como citar este artigo:

Dias, C; Verona, A. Excesso de

peso, obesidade e educação no Brasil. Revista Saúde (Sta. Maria).

2019; 45 (2).

\section{Autor correspondente:}

Nome: Claudio Santiago Dias Junior

E-mail: csdj@ufmg.br

Telefone: (3 I) 34093833

Formação Profissional: Doutor em

Demografia pela Universidade

Federal de Minas Gerais (UFMG),

Belo Horizonte, MG, Brasil.

\section{Filiação Institucional:}

Departamento de Sociologia (UFMG)

Endereço para correspondência:

Rua: Castelo Guimaräes n :

447/202 Bairro: Castelo

Cidade: Belo Horizonte

Estado: Minas Gerais

CEP: $31330-250$

Data de Submissão:

02/05/2018

Data de aceite:

26/05/2019

Conflito de Interesse: Não há conflito de interesse

\section{(cc) $\mathrm{BY}-\mathrm{NC}-\mathrm{ND}$}

\author{
Claudio Santiago Dias Jr, Ana Paula Verona
}

\section{RESUMO}

Este estudo tem como objetivo descrever a prevalência do excesso de peso e da obesidade no Brasil em 2006 e 2016, segundo o sexo e escolaridade e verificar se a escolaridade ainda é um fator de proteção para as mulheres, e se entre os homens, o paradoxo escolaridade/obesidade ainda permanece. Para isso, este trabalho utilizou as informações da pesquisa "Vigilância de Fatores de Risco e Proteção para Doenças Crônicas por Inquérito Telefônico" (VIGITEL) dos anos de 2006 e 2016. Ao contrário das evidências recentes encontradas nos EUA e Europa, a educação parece não ter um papel protetor para os homens brasileiros no que se refere ao excesso de peso e obesidade. Em relação às mulheres, essa variável ainda é importante, pois parece ter um papel protetor para 0 ganho de peso, embora já seja possível identificar que mesmo entre as mais escolarizadas, o aumento da prevalência do excesso de peso e da obesidade no período ocorreu.

PALAVRAS-CHAVE: Obesidade, Excesso de peso, Brasil, Vigitel.

\section{ABSTRACT}

The aim of this study is to describe the prevalence of overweight and obesity in Brazil in 2006 and 2016, according to gender and education and to verify if education is still a protective factor for women, and if among men, the paradox education / obesity still remains. This study used the information from the survey "Surveillance of Risk Factors and Protection for Chronic Diseases by Telephone Survey" (VIGITEL) from 2006 and 2016. Contrary to recent evidence found in the US and Europe, education does not appear play a protective role for Brazilian men regarding overweight and obesity. Regarding women, this variable is still important, as it seems to have a protective role for weight gain, although it is already possible to identify that even among the most educated, the increased prevalence of overweight and obesity in the period.

KEYWORDS: Obesity, Overweight, Brazil, Vigitel. 


\section{INTRODUÇÃO}

A obesidade tornou-se como um grave problema de saúde pública. Estima-se em 700 milhões o número de pessoas obesas no mundo, e projeções realizadas para o ano de 2030 apontam para um cenário ainda mais preocupante, onde esse contingente pode ultrapassar a barreira de 1,2 bilhões de pessoas ${ }^{1}$. As explicações para a chamada pandemia da obesidade vão desde o desequilíbrio entre a ingestão e gasto de calorias pelos indivíduos, passando pelos fatores genéticos, socioeconômicos, ambientais e individuais, que podem afetar a patogênese da obesidade ${ }^{2,8}$. Outro ponto importante para a explosão da obesidade pode ser a transformação observada na indústria alimentícia, com seus produtos ultra processados com apelos de saúde e a influência do agronegócio, em busca de novos consumidores para seus alimentos industrializados ${ }^{1}$.

Estudos evidenciam que a pandemia da obesidade está concentrada nos países mais pobres. Cerca de $60 \%$ dos indivíduos obesos vivem atualmente em países em desenvolvimento, principalmente na América Central, Oriente Médio e nas ilhas do pacífico e caribe. Nos países mais ricos a obesidade se concentra, principalmente, entre os moradores das áreas mais pobres e degradadas das grandes cidades e nas áreas rurais ${ }^{1,4,9}$.

Ao contrário de outros fatores de risco para a saúde, como o uso do tabaco e a desnutrição infantil, a incidência da obesidade está aumentando, e com isto, também aumenta a incidência de doenças como o câncer, diabetes, hipertensão, acidente vascular cerebral e doenças cardiovasculares, doenças renais crônicas, dentre outras. Tal situação faz com que a pandemia da obesidade se torne o maior desafio para as políticas públicas de saúde ${ }^{9,11}$.

O caso brasileiro é emblemático pela rapidez com que o excesso de peso e a obesidade avançam no país. Em um curto espaço de tempo, o Brasil deixou de ser um país de subnutridos para se tornar um país de mal nutridos com excesso de peso, com um aumento consistente no diagnóstico médico de diabetes, hipertensão, doenças cardiovasculares, doenças renais e câncer ${ }^{3,12,14}$.

Um aspecto relevante observado no caso brasileiro é a associação entre variáveis socioeconômicas, em especial a escolaridade, e obesidade. Existe uma relação positiva entre escolaridade e obesidade para os homens e uma relação negativa entre escolaridade e obesidade para as mulheres no Brasil. Tal fenômeno reforça a ideia de que a escolaridade pode ser um fator protetivo para as mulheres brasileiras em relação ao ganho de peso, não tendo o mesmo impacto para os homens ${ }^{15}$.

Diante da importância que a obesidade tem hoje não apenas na saúde pública, como também nos mais diversos setores da visa social, este estudo tem como objetivo principal descrever a prevalência do excesso de peso e da obesidade no Brasil em 2006 e 2016, segundo o sexo e escolaridade e verificar se a escolaridade ainda é um fator de proteção 
para as mulheres, e se entre os homens, o paradoxo escolaridade/obesidade ainda permanece. Para isso, este trabalho utilizará as informações da pesquisa "Vigilância de Fatores de Risco e Proteção para Doenças Crônicas por Inquérito Telefônico" (VIGITEL) dos anos de 2006 e 2016.

\section{DADOS E MÉTODO}

Os dados utilizados neste trabalho foram retirados da pesquisa VIGITEL dos anos de 2006 e 2016. Esta pesquisa coleta informações socioeconômicas e demográficas, características do padrão de alimentação e atividades físicas, associadas à ocorrência de doenças crônicas não transmissíveis (DNCT) e características de saúde e hábitos saudáveis de vida dos indivíduos a partir de um inquérito telefônico. Esta pesquisa ocorre anualmente desde 2006, é coordenada pelo Ministério da Saúde e seus dados estão disponibilizados para o público.

O banco de dados é formado por homens e mulheres com 18 anos e mais de idade, selecionados a partir de uma amostra probabilística. Em 2006 foram coletadas informações de 54.369 indivíduos (21.294 homens e 33.075 mulheres), e em 2016 foram coletadas informações de 53.210 indivíduos (20.258 homens e 32.952 mulheres). 0 campo foi realizado nas capitais dos 26 estados brasileiros mais o Distrito Federal.

Para a realização deste estudo foram utilizadas as seguintes informações:

1- Índice de Massa Corporal (IMC) (excesso de peso $\geq 25 \mathrm{Kg} / \mathrm{m}^{2}$ e obesidade $\geq 30 \mathrm{Kg} / \mathrm{m}^{2}$ ),

2- $\quad$ Sexo (masculino e feminino),

3- $\quad$ Escolaridade ( 0 a 8, 9 a 11 e 12 anos e mais de estudo).

É importante ressaltar que os cálculos do IMC foram realizados a partir das informações sobre peso e altura recordados pelos indivíduos selecionados na amostra, e as informações sobre anos de escolaridade foram agrupadas segundo os ciclos educacionais brasileiros.

Todas as análises descritivas realizadas neste estudo foram feitas buscando explicitar as possíveis associações entre excesso de peso/obesidade, sexo e escolaridade para os anos de 2006 e 2016.

\section{RESULTADOS}

Os resultados da pesquisa VIGITEL 2006 e 2016 mostram que no Brasil houve um aumento, em pontos percentuais, de indivíduos com 18 anos e mais de idade com excesso de peso (43,2\% para 53,8\%) e obesidade (11,6\% para 18,9\%) no período de 10 anos. Ao estratificar estes resultados segundo sexo (GRÁFICO 1), evidencia-se uma maior 
prevalência de sobrepeso entre os homens, tanto em 2006 quanto em 2016, e uma maior prevalência de obesidade entre as mulheres no mesmo período. Um fato que chama a atenção é que entre as mulheres o aumento, em pontos percentuais, foi maior que entre os homens, tanto no que se refere ao excesso de peso $(7,5 \%$ contra $6,7 \%)$ quanto à obesidade ( $12 \%$ contra $10,5 \%)$.

Gráfico 1 - Prevalência do excesso de peso e obesidade (\%), segundo sexo, Brasil, 2006-2016

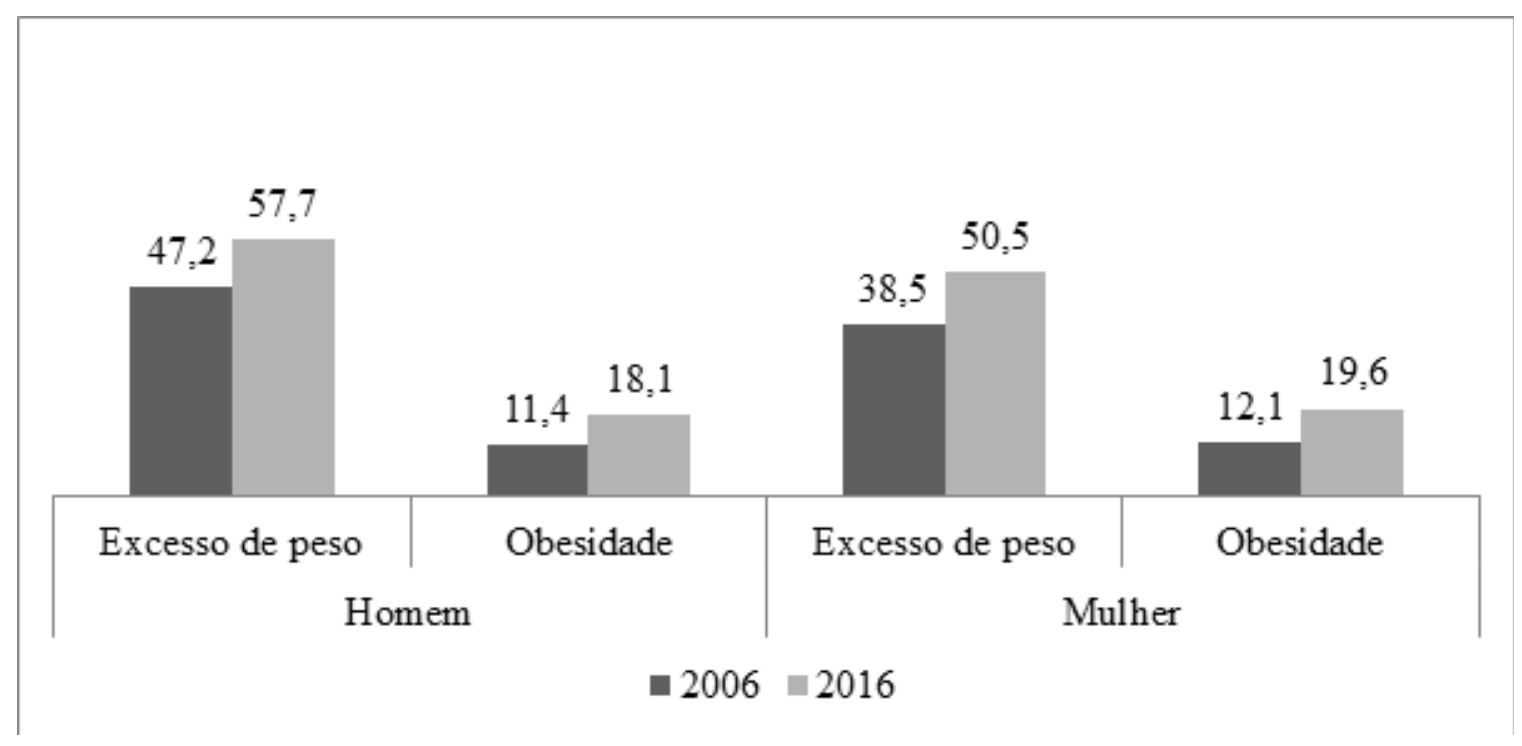

Fonte: VIGITEL, 2006, 2016.

Ao desagregar os dados segundo sexo e educação para analisar a prevalência do excesso de peso e obesidade (Tabela 1), são claras as diferenças entre homens e mulheres. Para as mulheres, quanto maior a escolaridade menor a prevalência do excesso de peso, fato observado tanto em 2006 quanto em 2016. Para os homens o comportamento é um pouco diferente. Nos dois períodos analisados, a maior prevalência do excesso de peso em ter a população masculina é sempre entre os grupos mais escolarizados, e a menor prevalência ocorre sempre no grupo de escolaridade intermediária.

Ainda de acordo com a Tabela 1, as mulheres tiveram os maiores ganhos percentuais no que se refere à prevalência de excesso de peso, em todos os três subgrupos educacionais. Entre as mulheres de escolaridade média (9-11) o acréscimo chegou a quase $20 \%$ e entre as mais escolarizadas quase $12 \%$. Comparando os grupos segundo 0 sexo, as mulheres com escolaridade intermediária e superior escolarizados tiveram um ganho percentual foi em torno de duas vezes maior que ao observado entre os homens.

Tabela 1 - Prevalência do excesso de peso e obesidade, segundo sexo e anos de estudo, Brasil, 2006-2016. 


\begin{tabular}{ccccccc}
\hline \multirow{2}{*}{ Anos de estudo } & \multicolumn{7}{c}{ Excesso de peso } \\
\cline { 2 - 7 } & \multicolumn{7}{c}{ Homem } & \multicolumn{5}{c}{ Mulher } \\
& 2006 & 2016 & $\neq$ & 2006 & 2016 & $\neq$ \\
$0-8$ & 45,9 & 58,0 & 12,1 & 47,2 & 60,3 & 13,1 \\
$9-11$ & 45,6 & 56,1 & 10,5 & 31,0 & 50,8 & 19,8 \\
$12+$ & 53,4 & 59,3 & 5,9 & 28,4 & 40,6 & 12,2 \\
\hline \multirow{7}{*}{ Anos de estudo } & Homem & \multicolumn{7}{c}{ Obesidade } \\
\cline { 2 - 7 } & 2006 & 2016 & $\neq$ & 2006 & 2016 & $\neq$ \\
\hline $0-8$ & 12,0 & 19,6 & 7,6 & 15,2 & 26,9 & 11,7 \\
$9-11$ & 9,9 & 17,2 & 7,3 & 8,1 & 19,4 & 11,3 \\
$12+$ & 11,8 & 17,7 & 5,9 & 7,5 & 12,8 & 5,3
\end{tabular}

Fonte: VIGITEL, 2006, 2016.

Os dados apresentados na Tabela 1 evidenciam um grande aumento na prevalência da obesidade no Brasil, em todos os subgrupos educacionais, independentemente do sexo. Em todos eles, o ganho em pontos percentuais foram maiores nos grupos de baixa e média escolaridade.

Entre as mulheres, ainda se percebe, nos dois períodos estudados, que quanto maior a escolaridade menor a prevalência da obesidade, sendo que a diferença entre os grupos de escolaridade, em pontos percentuais, aumentou entre 2006 e 2016. Para exemplificar, a diferença em pontos percentuais entre mulheres com escolaridade média e escolaridade alta que era de 0,6 em 2006 passa para 6,6 pontos percentuais em 2016. Por outro lado, a diferença entre as mulheres com menos escolaridade em relação às mulheres com mais escolaridade passou de $7,7 \%$ para $6,4 \%$, uma redução de 1.3 pontos percentuais.

Entre os homens mais escolarizados, a variação no período foi menor, se comparado aos demais grupos. Para os homens com 12 anos ou mais de escolaridade o aumento na prevalência da obesidade foi de 5,8 pontos percentuais, ao passo que entre os homens com 0 e 8 anos de escolaridade 0 aumento foi de 7,6 pontos percentuais.

Se observarmos os grupos extremos de educação (0-8 e 12+), a diferença, em pontos percentuais, entre os homens é de 2,1 e entre as mulheres 14,1 no ano de 2016. Ou seja, pode-se admitir que a prevalência da obesidade entre os homens é mais homogênea que entre as mulheres, segundo os grupos educacionais.

\section{DISCUSSÃO}

Os resultados deste trabalho confirmam a situação gravíssima que passa o Brasil em relação ao rápido ganho de peso da população. Mais de $50 \%$ da população está com excesso de peso e quase $20 \%$ estão obesos. 
Como observado em estudos prévios ${ }^{1,9}$ a maior prevalência do excesso de peso ocorre entre os homens, e a obesidade é mais comum entre as mulheres, embora, no caso brasileiro, em ambos os casos a situação seja preocupante.

Ao contrário das evidências recentes encontradas nos EUA e Europa, a educação parece não ter um papel protetor para os homens brasileiros no que se refere ao excesso de peso e obesidade ${ }^{1}$. Em relação às mulheres, essa variável ainda é importante, pois parece ter um papel protetor para o ganho de peso, embora já seja possível identificar que mesmo entre as mais escolarizadas, o aumento da prevalência do excesso de peso e da obesidade no período ocorreu. Ou seja, a escolaridade entre as mulheres ainda parece ser um fator importante para o ganho de peso, mas pode estar perdendo força. Já entre os homens, dada a homogeneidade na distribuição do excesso de peso e obesidade, segundo grupos de escolaridade, a educação pode não ser um fator decisivo de proteção.

\section{CONSIDERAÇÕES FINAIS}

Os resultados apresentados por este estudo são importantes porque podem auxiliar no entendimento da pandemia da obesidade no Brasil. Já é consenso que o excesso de peso da população traz consequências negativas tanto para o indivíduo quanto para a sociedade, com impactos expressivos no sistema público de saúde. Ações são demandadas pela sociedade, e a resposta do poder público parece não surtir os efeitos esperados, dado o sistemático aumento observado, ano a ano, da prevalência do sobrepeso e obesidade no Brasil.

Saber como o sobrepeso e obesidade se distribui entre os subgrupos específicos da sociedade pode possibilitar ações mais focalizadas que podem produzir resultados mais efetivos no combate à pandemia da obesidade.

Embora seja ainda um trabalho inicial, os resultados deste estudo mostram que a educação pode ter um papel importante no controle de peso entre as mulheres, mas pode estar perdendo a capacidade de protegê-las do ganho de peso. Ao mesmo tempo pode ser que entre os homens a educação não tenha o mesmo efeito.

Novos estudos são necessários para identificar possíveis relações entre variáveis socioeconômicas e obesidade para um melhor desenvolvimento de políticas públicas de combate a essa pandemia.

\section{REFERÊNCIAS}

1. Popkin, BM, Adair, LS. NG, SW. Global nutrition transition and the pandemic of obesity in developing countries. Nutr Rev, [internet] 2012 [acesso em 2018 mar 14] Jan;70(1):3-21. Disponível em: http://onlinelibrary.wiley.com/ doi/10.1111/j.1753-4887.2011.00456.x/abstract 
2. Bray, GA. Kim, JPH. Obesity: a chronic relapsing progressive disease process. A position statement of the World Obesity Federation. Obes Rev, [internet] 2017 [acesso em 2018 mar 14]; v.18,715-723.Disponível em: http://onlinelibrary.wiley.com/doi/10.1111/obr.12551/epdf

3. Castro, IRR. Obesidade: urge fazer avançar políticas públicas para sua prevenção e controle. Cad Saúde Pública,[internet] 2017 [acesso em 2018 mar 14]; 33 (7):e00100017. Disponível em: http://www.scielo.br/pdf/csp/v33n7/ 1678-4464-csp-33-07-e00100017.pdf

4. Smith, KB, Smith, MS. Obesity Statistics. Prim Care, [internet] 2016 [acesso em 2018 mar 14] Volume 43, Issue 1,121-135. Disponível em: http://www.primarycare.theclinics.com/article/S0095-4543(15)00098-6/pdf

5. Le Blanc, A. Why are children sedentary: an examination using the International Study of Childhood Obesity, Lifestyle and the Environment. Appl Physiol Nutr Metab, [internet] 2016 [acesso em 2018 mar 14]; 41(7):790-790. Disponível em: https://www.ncbi.nlm.nih.gov/pubmed/27266396

6. Vilchis-Gil J, Galván-Portillo M, Klünder-Klünder M et al. Food habits, physical activities and sedentary lifestyles of eutrophic and obese school children: a case-control study. BMC Public Health,[internet] 2015 [acesso em 2018 mar 14] 15: 124. Disponível em: https://bmcpublichealth.biomedcentral.com/track/pdf/10.1186/s12889-015-1491-1?site=bmcpublichealth.biomedcentral.com

7. Faghri, P. Stratton, K. Momeni, K. Sedentary Lifestyle, Obesity, and Aging: Implication for Prevention J Nutr Disord Ther, [internet] 2015[acesso em 2018 mar 14]; 5:e119. Disponível em: https://www.omicsonline.org/open-access/ sedentary-lifestyle-obesity-and-agingimplication-for-prevention-2161-0509-5-e119.php?aid=37181

8. Bray, GA, Popkin, BM. Dietary fat intake does affect obesity. Am J Clin Nutr [internet] 1998 [acesso em 2018 mar 14]; v.68, 1157-1173. Disponível em: http://www.cpc.unc.edu/projects/nutrans/publications/Bray-Popkin-AJCN.pdf

9. NG, M, Fleming, T, Robinson,M. et al. Global, regional, and national prevalence of overweight and obesity in children and adults during 1980-2013: a systematic analysis for the Global Burden of Disease Study 2013. Lancet, [internet] 2014 [acesso em 2018 mar 14] Volume 384 , Issue 9945, 766 - 781. Disponível em: http://www.thelancet.com/pdfs/ journals/lancet/PIIS0140-6736(14)60460-8.pdf 
10. Dias, PC, Henriques, P, Anjos, LA. et al. Obesidade e políticas públicas: concepções e estratégias adotadas pelo governo brasileiro. Cad. Saúde Pública, [internet] 2017 [acesso em 2018 mar 14]; 33 (7): e00006016. Disponível em: http://www.scielo.br/pdf/csp/v33n7/en_1678-4464-csp-33-07-e00006016.pdf

11. Wannmacher, L. Obesidade como fator de risco para morbidade e mortalidade: evidências sobre o manejo com medidas não medicamentosas. Uso Racional de Medicamentos: fundamentação em condutas terapêuticas e nos macroprocessos da Assistência Farmacêutica,[[internet] 2016 [acesso em 2018 mar 14] v. 1, n. 7, 2016. Disponível em:http://www.paho.org/bra/index.php?option=com_docman\&view=download\&alias=1535-obesidade-como-fator-risco-para-morbidade-e-mortalidade-evidencias-sobre-0-manejo-com-medidas-nao-medicamentosas-5\&category_slug=serie-uso-racional-medicamentos-284\&ltemid=965

12. Vigitel Brasil 2016 Vigilância de fatores de risco e proteção para doenças crônicas por inquérito telefônico. [internet] 2016 [acesso em 2018 fev 6] Disponível em: http://portalarquivos.saude.gov.br/images/pdf/2017/junho/07/vigitel_2016_jun17.pdf.

13. Malta, DC, Andrade, SC, Claro, RM, et al. Evolução anual da prevalência de excesso de peso e obesidade em adultos nas capitais dos 16 estados brasileiros e no Distrito Federal entre 2006 e 2012. Rev Bras Epidemiol, [internet] 2014 [acesso em 2018 mar 14] Suplemento PeNSE, 267-276. Disponível em: http://www.scielo.br/pdf/rbepid/v17s1/ pt_1415-790X-rbepid-17-s1-00267.pdf

14. Lima, NP, Horta, BL, Motta, JVS et al. Evolução do excesso de peso e obesidade até a idade adulta, Pelotas, Rio Grande do Sul, Brasil, 1982-2012. Cad. Saúde Pública, [internet] 2015 [acesso em 2018 mar 14]; 31(9), 2017-2025. Disponível em: http://www.scielo.br/pdf/csp/v31n9/0102-311X-csp-31-9-2017.pdf

15. Andrade, RG, Chaves, OC, Costa, et al. Excesso de peso em homens e mulheres residentes em área urbana: fatores individuais e contexto socioeconômico. Cad. Saúde Pública [internet] 2015 [acesso em 2018 mar 14]; 31(Suppl. 1), 148-158. Disponível em: https://www.scielosp.org/article/ssm/content/raw/?resource_ssm_path=/media/assets/ csp/v31s1/pt_0102-311X-csp-31-s1-0148.pdf 